\title{
Inelastic Stress Analysis of Curved Beams with Bending and Shear Coupling
}

\author{
Yen-Po Wang ${ }^{1}$, Chien-Liang Lee ${ }^{2}$, Shih-Chiao Huang ${ }^{1}$ \\ ${ }^{1}$ Dept. Civil Engineering/National Chiao-Tung University \\ 1001 Ta-Hsueh Road, Hsinchu, Taiwan \\ ypwang@mail.nctu.edu.tw; joejoe9193.cv03g@mail.nctu.edu.tw \\ ${ }^{2}$ School of Civil Engineering and Architecture/Xiamen University of Technology \\ No. 600 Ligong Road, Jimei District, Xiamen, 361024, Fujian Province, China \\ cllee63@ hotmail.com
}

\begin{abstract}
The inelastic stress analysis of curved beams under pure bending has been extensively studied in the past few years. Nevertheless, the analysis for those under end shear, or moment and shear coupling, are not yet available. In this paper, the analytical model for inelastic stress analysis of symmetrical curved beams with bending and shear coupling is derived. This problem arises from the investigation of the inelastic behaviour of an in-plane fle xu ral damper consisting of a circular arch with two straight arms. In-plane lateral forces applying to the end of the arm makes the arch a circular cantilever subjected to moment and shear simultaneously. Extended from the classical theory of elasticity, the proposed computational model adopts the generalized Hook's law in plane stress with consideration of the plastic strains defined by the total deformation theory. The swift-type nonlinear hardening law is considered for the inelastic constitutive relations of the material with its behaviour governed by von Mises' yield criterion. With the introduction of a certain form of Airy stress function that satisfies the compatibility equation in polar coordinates, the strain -compatibility equation with the corresponding boundary conditions can be simplified as a second-order ordinary differential equation of a properly defined generalized stress function dependent on radius of the wide curved beam. Solution of this ODE equation becomes a boundary -valued problem that can be solved numerically in both elastic and inelastic stages. To validate the proposed model, a preliminary numerical study of the stress analysis in the elastic stage has been conducted. Encouragingly, the numerical solution agrees perfectly with its analytical counterpart given by classical theory of elasticity.
\end{abstract}

Keywords: inelastic stress analysis, elasticity, curved beam, total deformation theory, strain-compatibility, boundaryvalued problem

\section{Introduction}

Supplemental energy-dissipative dampers have been widely adopted for earthquake-protection of building structures. Metallic yielding dampers such as ADAS[1], TADAS[2] or Pre-bent stripe[3] that utilize the strength and ductility of general steel plates are alternatives considered to be cost-effective among others. The aforementioned metallic dampers are in common designed to deform in an out-of-plane flexural mode by resisting the loading with the ir weak axis. As an effort to improve material utilization, seismic structural dampers designed to deform inelastically in an in-plane flexural mode have attracted serious attentions recently[4-7]. Preliminary component test of an arch-shaped damper suggests its potential in practical use with a drastic improvement in ultimate strength and ductility by considerably reducing the effect of warping and stress concentration. To get more insight of the elastic-plastic behaviour of the in-plane arch-shaped damper, a comprehensive inelastic stress analysis under monotonic load is desired for design purposes.

Eraslan and Arslan[8] proposed a concise analytical model for the elastic-plastic stress analysis of a strain hardening curved beam under pure bending with solutions for both plane stress and plane strain conditions according to Tresca's yie lding criterion. Eraslan and Arslan[9] conducted also a computational study on the inelastic stress analysis of nonlinear hardening curve beam under pure bending in plane stress using von Mises' yielding criterion. With the loading restrained to pure bending only, their studies provide a sound and comprehensive framework for inelastic stress analysis of curved beams with strain hardening material. In-plane lateral forces applying to the end of the arch-shaped damper makes it a circular cantilever subjected to moment and shear simultaneously. Under these circumstances the model derived by Eraslan and Arslan[8,9] is no longer valid and the corresponding analytical models need to be developed. Extended from the 
classical theory of elasticity, the proposed computational model adopts the generalized Hook's law in plane stress with consideration of the plastic strains defined by the total deformation theory. The swift-type nonlinear hardening law is considered for the inelastic constitutive relations of the material with its behaviour governed by von Mises' yield criterion. With the introduction of a certain form of Airy stress function that satisfies the compatibility equation in polar coordinates, the strain-compatibility equation with the corresponding boundary conditions can be simplified as a second-order ordinary differential equation of a properly defined generalized stress function dependent on radius of the wide curved beam. Solution of this ODE equation becomes a boundary-valued problem that can be solved numerically in both elastic and ine lastic stages. To validate the proposed model inspired by the work of Eraslan and Arslan [8, 9], a preliminary numerical study of the stress analysis in the elastic stage has been conducted. Encouragingly, the numerical solution matches perfectly with its analytical counterpart given by classical theory of elasticity [10].

\section{Computational Model}

An in-plane flexural damper under a load $2 P$ at a distance $l$ from the ends of the circular arch is illustrated in Fig. 1(a). The ends of the arch are subjected to a bending moment $M=P l$ and a shear $P$ simultaneously, as indicated in Fig. 1(b). Taking advantage of symmetry with respect to the vertical axis, the task can be reduced as solving a problem of a circular cantilever subjected to coupling of shear and moment at its end, as indicated in Fig. 1(c).

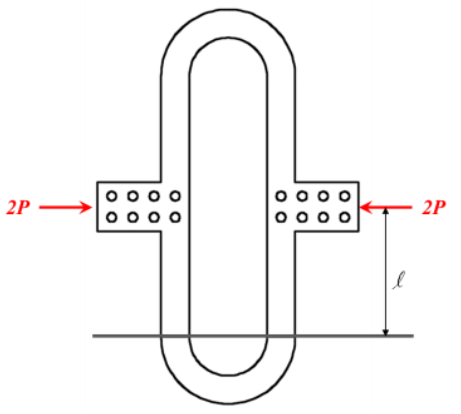

(a) in-Plane Flexural Damper

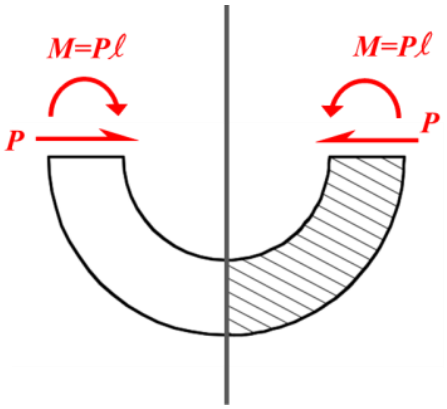

(b) Free-body of the Circular Arch

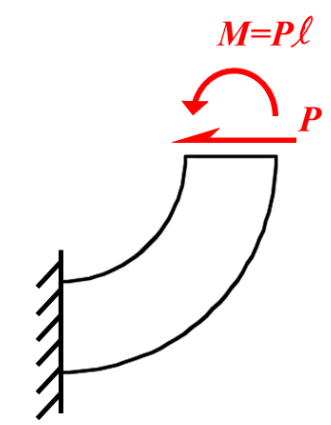

(c) Circular Cantilever

Fig. 1: The Damper and the Portion Considered in Analysis .

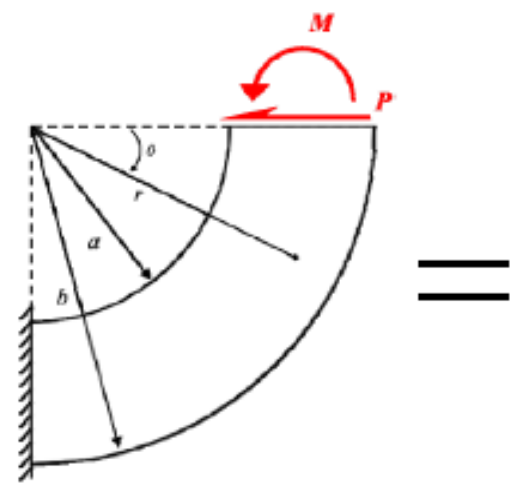

(a) P-M Coupling

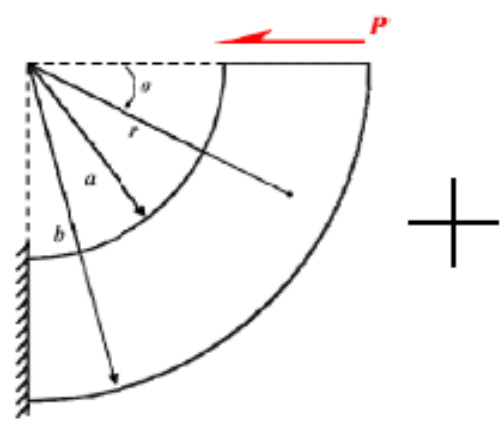

(b) End-Shear (P) only

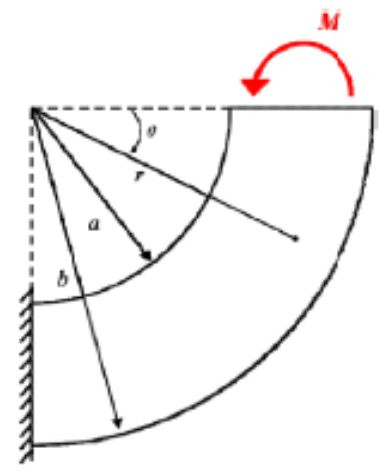

(c) Pure Bending (M)

Fig. 2: Resolving Diagram of the Loads on the Circular Cantilever.

\subsection{Fundamental of Solid Mechanics}

The curved beam with inner radius $a$ and outer radius $b$ is considered, as shown in Fig. 2. It is most convenient to start with the well-developed fundamentals of elasticity in polar coordinates [10]. For plane stress problems, the equilibrium equations of the curved beam with a constant thickness $\left(t_{z}\right)$ can be expressed as 


$$
\begin{gathered}
\frac{\partial \sigma_{r}}{\partial r}+\frac{1}{r} \frac{\partial \tau_{r \theta}}{\partial \theta}+\frac{\sigma_{r}-\sigma_{\theta}}{r}=0 \\
\frac{\partial \tau_{r \theta}}{\partial r}+\frac{1}{r} \frac{\partial \sigma_{\theta}}{\partial \theta}+\frac{2 \tau_{r \theta}}{r}=0
\end{gathered}
$$

Where $\sigma_{r}, \sigma_{\theta}$ are respectively the normal stress in the radial and tangential directions, and $\tau_{r \theta}$ is the shear stress. These stress components can be further written in terms of an Airy stress function $\varphi(r, \theta)$ as

$$
\begin{gathered}
\sigma_{r}=\frac{1}{r} \frac{\partial \varphi}{\partial r}+\frac{1}{r^{2}} \frac{\partial^{2} \varphi}{\partial \theta^{2}} \\
\sigma_{\theta}=\frac{\partial^{2} \varphi}{\partial r^{2}} \\
\tau_{r \theta}=-\frac{\partial}{\partial r}\left(\frac{1}{r} \frac{\partial \varphi}{\partial \theta}\right)
\end{gathered}
$$

Which satisfy the equilibrium equation (1). The strains are related to the displacement fields $(u, v)$ respectively for the radial and tangential displacements as

$$
\begin{gathered}
\varepsilon_{r}=\frac{\partial u}{\partial r} \\
\varepsilon_{\theta}=\frac{u}{r}+\frac{1}{r} \frac{\partial v}{\partial \theta} \\
\gamma_{r \theta}=\frac{1}{r} \frac{\partial u}{\partial \theta}+\frac{\partial v}{\partial r}-\frac{v}{r}
\end{gathered}
$$

where $\varepsilon_{r}, \varepsilon_{\theta}$ are respectively the normal strain in the radial and tangential directions, and $\gamma_{r \theta}$ is the shear strain. The strain-compatibility equation according to [11] is expressed as

$$
\frac{\partial}{\partial r}\left(r \frac{\partial \gamma_{r \theta}}{\partial \theta}-r^{2} \frac{\partial \varepsilon_{\theta}}{\partial r}\right)+r \frac{\partial \varepsilon_{r}}{\partial r}-\frac{\partial^{2} \varepsilon_{r}}{\partial \theta^{2}}=0
$$

And the compatibility can further be expressed in terms of the Airy stress function $\varphi(r, \theta)$ as

$$
\left(\frac{\partial^{2}}{\partial r^{2}}+\frac{1}{r} \frac{\partial}{\partial r}+\frac{1}{r^{2}} \frac{\partial^{2}}{\partial \theta^{2}}\right)\left(\frac{\partial^{2} \varphi}{\partial r^{2}}+\frac{1}{r} \frac{\partial \varphi}{\partial r}+\frac{1}{r^{2}} \frac{\partial^{2} \varphi}{\partial \theta^{2}}\right)=0
$$

The von Mises' yielding criterion adopted in this study is defined as

$$
\sigma_{v M}=\sqrt{\sigma_{r}^{2}-\sigma_{r} \sigma_{\theta}+\sigma_{\theta}^{2}+3 \tau_{r \theta}} \geq \sigma_{y}
$$

And the material becomes partially plastic wherever the inequality holds. The generalized Hooke's law is written in accordance with the total deformation theory [12] as 


$$
\begin{gathered}
\varepsilon_{r}=\varepsilon_{r}^{P}+\frac{1}{E}\left(\sigma_{r}-v \sigma_{\theta}\right) \\
\varepsilon_{\theta}=\varepsilon_{\theta}^{P}+\frac{1}{E}\left(\sigma_{\theta}-v \sigma_{r}\right) \\
\gamma_{r \theta}=\gamma_{r \theta}^{p}+\frac{\tau_{\gamma \theta}}{G}
\end{gathered}
$$

where the plastic strains are defined by

$$
\begin{gathered}
\varepsilon_{r}^{P}=\frac{\varepsilon_{E Q}}{\sigma_{v M}}\left(\sigma_{r}-\frac{1}{2} \sigma_{\theta}\right)=\frac{1}{E_{p}}\left(\sigma_{r}-\frac{1}{2} \sigma_{\theta}\right) \\
\varepsilon_{\theta}^{P}=\frac{\varepsilon_{E Q}}{\sigma_{v M}}\left(\sigma_{\theta}-\frac{1}{2} \sigma_{r}\right)=\frac{1}{E_{p}}\left(\sigma_{\theta}-\frac{1}{2} \sigma_{r}\right) \\
\gamma_{r \theta}^{p}=\frac{\tau_{r \theta}}{G_{p}}=\frac{3}{E_{p}} \tau_{r \theta}
\end{gathered}
$$

In which the equivalent plastic strain of a swift-type nonlinear hardening law is considered as

$$
\begin{aligned}
\varepsilon_{E Q}=\frac{\left(\frac{\sigma_{v M}}{\sigma_{y}}\right)^{m}-1}{H} & \text { for } \sigma_{v M} \geq \sigma_{y} \\
=0 & \text { for } \quad \sigma_{v M}<\sigma_{y}
\end{aligned}
$$

Where parameters $m$ and $H$ characterize the post-yielding behaviour of the material.

\subsection{Pure Bending}

Under the condition of pure bending, as indicated in Fig. 2(c), the shear stress vanishes and the equilibrium equation reduces to

$$
\sigma_{\theta}=\frac{d}{d r}\left(r \sigma_{r}\right)
$$

If a generalized stress function $Y_{1}(r)$ is defined, according to Eraslan and Arslan [9], as

$$
Y_{1}(r)=r \sigma_{r}
$$

and, as a result

$$
\sigma_{\theta}=\frac{d Y_{1}(r)}{d r}
$$

Using the strain-displacement relations in eq.(3) and taking into account the fact of shear strain being zero, the strain-compatibility eq.(4) can be simplified as 


$$
\frac{\partial}{\partial r}\left(r \varepsilon_{\theta}\right)-\varepsilon_{r}=\phi_{M}
$$

where $\phi_{M}$ is an integration constant. Substitution of the generalized Hooke's law (7) for the strains and expressing the stresses in terms of the generalized stress function, $Y_{1}$, Eq.(13) can be written in the form of a second-order ordinary differential equation of $Y_{1}$ as

$$
r^{2} Y_{1}^{\prime \prime}+r Y_{1}^{\prime}-Y_{1}=r\left(\phi_{M}+\varepsilon_{r}^{p}-\varepsilon_{\theta}^{p}-r \frac{d \varepsilon_{\theta}^{p}}{d r}\right)
$$

or in brief

$$
Y_{1}^{\prime \prime}=F\left(r, Y_{1}, Y_{1}^{\prime}\right)
$$

With the following boundary conditions

$$
\begin{gathered}
Y_{1}(a)=Y_{1}(b)=0 \\
\int_{a}^{b} Y_{1}^{\prime} r d r=-M / t_{z}=-P l / t_{z}
\end{gathered}
$$

The $4^{\text {th }}$-order Runge-Kutter algorithm is adopted for solving the ODE problem iteratively along with Newton's method in the shooting process to accelerate convergence of the boundary-valued problem, as suggested by Eraslan and Arslan [9]. Once $Y_{1}$ is solved, the stress components can be found by eqs.(11) and (12) immediately.

\subsection{End Shear}

With a shear force acting at the end of the curved beam, as indicated in Fig. 2(b), the condition becomes more complicated with the presence of shear stress and all the stress components are now dependent on both $r$ and $\theta$. However, if we loan of the Airy stress function $\varphi(r, \theta)$ in the form of

$$
\varphi(r, \theta)=f(r) \sin \theta
$$

From the classical theory of elasticity [10] for the very same problem to start with, then the stress components can be written as

$$
\begin{gathered}
\sigma_{r}=\left(\frac{1}{r} f^{\prime}-\frac{1}{r^{2}} f\right) \sin \theta \\
\sigma_{\theta}=f^{\prime \prime} \sin \theta \\
\tau_{r \theta}=-\left(\frac{1}{r} f^{\prime}-\frac{1}{r^{2}} f\right) \cos \theta
\end{gathered}
$$

Let's define another generalized stress function $Y_{2}(r)$ as

$$
Y_{2}(r)=f^{\prime}-\frac{f}{r}
$$


Then the stress components in eq. (18) can be written in terms of $Y_{2}(r)$ to be

$$
\begin{gathered}
\sigma_{r}=\frac{Y_{2}}{r} \sin \theta \\
\sigma_{\theta}=\left(Y_{2}^{\prime}+\frac{Y_{2}}{r}\right) \sin \theta \\
\tau_{r \theta}=\frac{-Y_{2}}{r} \cos \theta
\end{gathered}
$$

As a result, the generalized Hooke's law (7) can be modified as

$$
\begin{gathered}
\varepsilon_{r}=\varepsilon_{r}^{P}+\frac{1}{E}\left\lfloor\frac{Y_{2}}{r}-v\left(Y_{2}^{\prime}+\frac{Y_{2}}{r}\right)\right\rfloor \sin \theta \\
\varepsilon_{\theta}=\varepsilon_{\theta}^{P}+\frac{1}{E}\left\lfloor Y_{2}^{\prime}+\frac{Y_{2}}{r}-v \frac{Y_{2}}{r}\right\rfloor \sin \theta \\
\gamma_{r \theta}=\gamma_{r \theta}^{p}+\frac{1}{G}\left(-\frac{Y_{2}}{r}\right) \cos \theta
\end{gathered}
$$

Upon substitution of eq. (21) for the strains into the strain-compatibility equation (4) and integrating it with respect to the radius $r$ leads to

$$
r^{2} Y_{2}^{\prime \prime}+r Y_{2}^{\prime}-4 Y_{2}=\phi_{p}+\frac{E}{\sin \theta}\left(r \frac{\partial \gamma_{r \theta}^{p}}{\partial \theta}-r^{2} \frac{\partial \varepsilon_{\theta}^{p}}{\partial r}+r \varepsilon_{r}^{p}\right)
$$

or in brief

$$
Y_{2}^{\prime \prime}=F\left(r, Y_{2}, Y_{2}^{\prime}\right)
$$

With the following boundary conditions

$$
\begin{gathered}
Y_{2}(a)=Y_{2}(b)=0 \\
\int_{a}^{b} \frac{Y_{2}}{r} d r=-\frac{P}{t_{z}}
\end{gathered}
$$

The same procedure as described in Section 2.2 can be adopted to solve the boundary-valued problem of eqs. (23)(24). Once $Y_{2}$ is solved, the stress components can be found by eqs.(20) immediately for a given cross-section at $\theta$.

\subsection{Bending and Shear Coupling}

If the curved beam is subjected to the coupling of bending $(M)$ and shear $(P)$ at the end simultaneously, the stresses can be obtained by summing the results from pure bending and end shear derived independently as

$$
\sigma_{r}=\sigma_{r, 1}+\sigma_{r, 2}
$$




$$
\begin{gathered}
\sigma_{\theta}=\sigma_{\theta, 1}+\sigma_{\theta, 2} \\
\tau_{r \theta}=\tau_{r \theta, 2}
\end{gathered}
$$

where the subscript 1 denotes those obtained from pure bending and subscript 2 from end shear. Note that under the coupling loading condition, the resultant stresses from eqs. (25) should be used in checking the yie lding condition via the von Mises criterion (6).

\section{Numerical Verification}

As an effort to validate the proposed model, a preliminary numerical study of the stress analys is has been conducted in the elastic stage where analytical solutions are available for comparison. An in-plane flexural steel damper in a $U$ shape considered for component test earlier by the authors is adopted herein. The inner radius $(a)$ of the circular arch of the damper is $45 \mathrm{~mm}$ and the outer radius $(b)$ is $220 \mathrm{~mm}$. The steel plate is $25 \mathrm{~mm}$ in thickness and the effective length $(l)$ of the straight arm reads $235 \mathrm{~mm}$. Parameters of the material are summarized in the following: Young's modulus $E=200 \mathrm{GPa}$, Poisson's ratio $v=0.3$ and the yield stress $\sigma_{y}=250 \mathrm{MPa}$. A compression force $P$ is acting inwards from each end of the arms through pin connection resulting in a moment of $P l$ and an end shear of $P$ simultaneous ly on the bottom of the arch, as indicated in Fig. 2(a). The yield strength $P_{y}=39.75 \mathrm{kN}$ determined by setting the normal stress $\sigma_{\theta}(a, \pi / 2)=\sigma_{y}$ where initial yie lding of the damper takes place is considered as the applied load. Stress components normalized with respect to the yield stress, $\sigma_{y}$, are presented graphically for $\theta=\pi / 2$ and $\theta=\pi / 4$ in Figs. 4(a) and 4(b), respectively. The bar over the stress symbols in the figures indicates the normalized stress. The numerical solution agrees perfectly with its analytical counterpart given by classical theory of elasticity [10]. The normalized von Mises stress $\bar{\sigma}_{v M}=\sigma_{v M} / \sigma_{y}=1$ occurs at the cross-section of $\theta / 2$ on the inner surface $r=a$ under yield load $P_{y}$.

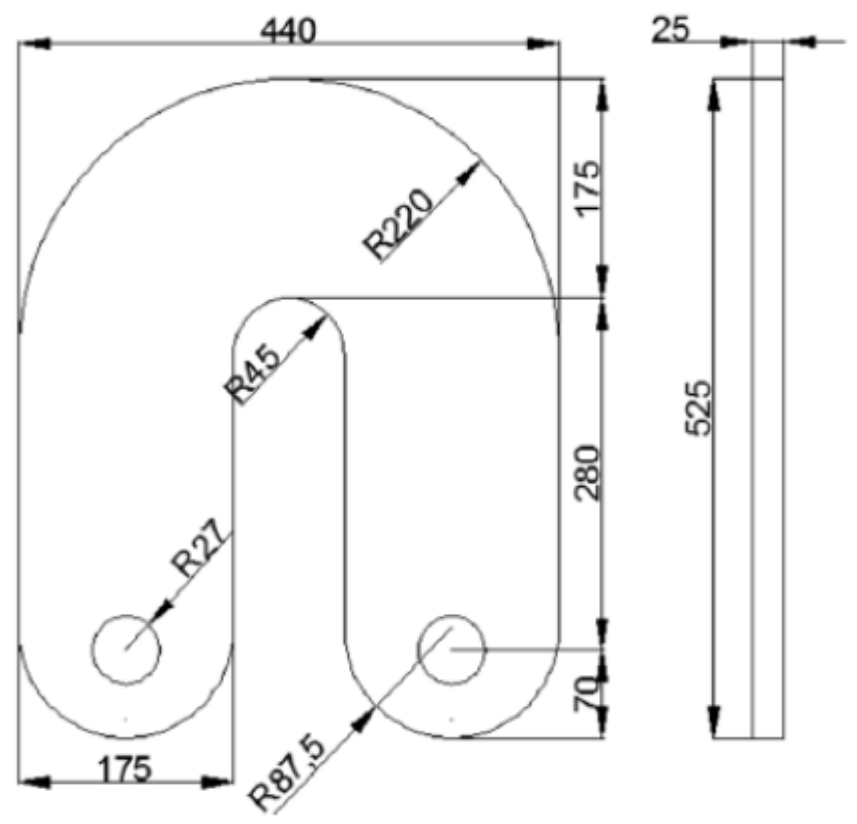

Fig. 3: Design Details of the in-Plane Flexural Damper Considered in the Numerical Example. 


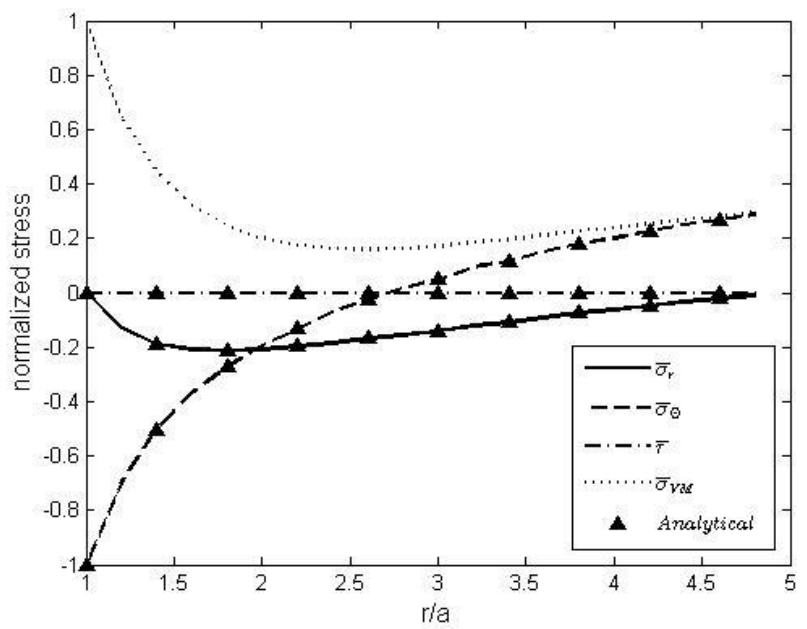

(a) $\theta$ a)/2

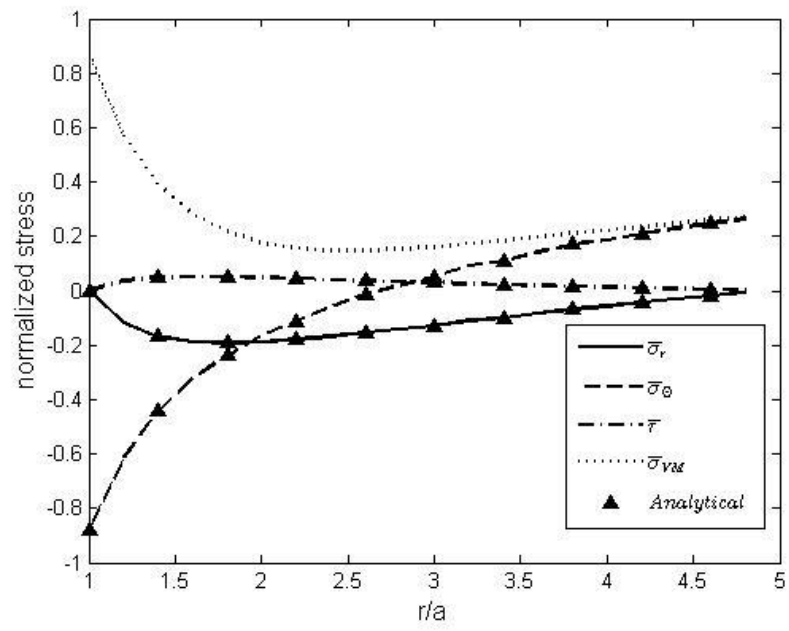

(b) $\theta=\pi / 4$

Fig. 4: Comparison of Numerical Solution in the Elastic Stage with the Analytical Counterpart $\left(P=P_{y}\right)$.

\section{Conclusion}

In this paper, the analytical model for inelastic stress analysis of symmetrical curved beams with bending and shear coupling represented in a second-order ordinary differential equation of a generalized stress function is derived. As an effort to validate the proposed model, a preliminary numerical study of the stress analysis in the elastic stage has been conducted. The numerical solution agrees perfectly with its analytical counterpart given by classical theory of elasticity. The model will be further used for the inelastic stress analysis of the in-plane flexural damper.

\section{Acknowledgements}

This research work is granted by the Ministry of Science and Technology of Republic of China under contract MOST 104-2221-E-009-197.

\section{References}

[1] A. S. Whittaker, V. V. Bertero, C. L. Thompson, and L. J. Alonso, "Seismic Testing of Steel Plate Energy Dissipation Devices," Earthquake Spectra, vol. 7, no. 4, pp. 563-604, 1991.

[2] K. C. Tsai, H. W. Chen, C. P. Hong, and Y. F. Su, "Design of Steel Triangular Plate Energy Absorbers for SeismicResistant Construction," Earthquake Spectra, vol. 9, no. 3, pp. 505-528, 1993.

[3] Y. P. Wang and C. S. Chang Chien, "A Study on Using Pre-Bent Steel Strips as Seismic Energy-Dissipative Devices," Earthquake Engineering \& Structural Dynamics, vol. 38, pp. 1009-1026, 2009.

[4] W. K. Chan and F. Albermani, "Experimental Study of Steel Slit Damper for Passive Energy Dissipation," Engineering Structures, vol. 30, pp. 1058-1066, 2008.

[5] J. H. Park and K. H. Lee, "Cyclic Loading Tests of Steel Dampers Utilizing Flexure-Analogy Deformation," in Proceedings of the $15^{\text {TH }}$ World Conference on Earthquake Engineering, Lisbon, Portugal, September 24-28, paper no. 1228, 2012.

[6] Z. Guan, J. Li, and Y. Xu, "Performance Test of Energy Dissipation Bearing and Its Application in Seismic Control of a Long-Span Bridge," Journal of Bridge Engineering, ASCE, vol. 15, pp. 622-630, 2010.

[7] Y. P. Wang, D. H. Chen, and C. L. Lee, "An Experimental Study on in-Plane Arch-shaped Flexural Damper," in Implementing Innovative Ideas in Structural Engineering and Project Management, Proceedings of ISEC-8, Nov. 2328, Parramatta, Australia, pp. 293-298, 2015.

[8] A. N. Eraslan and E. Arslan, "A Concise Analytical Treatment of Elastic-Plastic Bending of a Strain Hardening Curved Beam," ZAMM, vol. 88, no. 8, pp. 600-616, 2008.

[9] A. N. Eraslan and E. Arslan, "A Computational Study on the Nonlinear Hardening Curved Beam Problem," International J. Pure and Applied Mathematics, vol. 43, no. 1, pp. 129-143, 2008. 
[10] S. P. Timoshenko and J. N. Goodier, Theory of Elasticity, Third Ed. New York: McGraw-Hill, 1970.

[11] A. P. Boresi, K. P. Chong, and J. D. Lee, Elasticity in Engineering Mechanics, Third Ed., Hoboken, New Jersey: John Wiley and Sons, Inc., 2011.

[12] R. M. Jones, Deformation Theory of Elasticity. Blacksburg, Virginia: Bull Ridge Publishing, 2009. 